\title{
The Screening Tool for Autism in Two Year Olds can identify children at risk of autism
}

Stone WL, Coonrod EE, Turner LM, et al. Psychometric properties of the STAT for early autism screening. J Autism Dev Disord 2004;34:691-701.

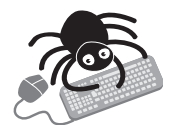

This article

contains extra text on the EBMH website

\section{How accurate is the Screening Tool for Autism in Two Year Olds (STAT) for identifying children at risk of autism?}

METHODS

$\square$

Design: Diagnostic cohort study

Setting: University based diagnostic evaluation centre and affiliated speech and hearing centre, Tennessee, USA; 1997 to 2001

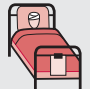

Patients: Initial validation of the STAT was carried out in 13 matched pairs of children aged 24-35 months, with a diagnosis of autism or alternatively developmental delay, language impairment, or both (DD/LI), and without severe sensory or motor impairment. Further validation was carried out in a sample of 104 children selected on the basis of parental report of developmental concerns.

Test: The STAT consists of 12 interactive items assessing different behavioural domains, including play, requesting, directing attention, and motor imitation. The test was administered by trained examiners who were blinded to the children's diagnoses. Overall score ranges from 0 to 4 , with lower scores indicating less impairment. The threshold for classification of being at "high risk" of autism was a score of 2 or above, based on initial calibration in 26 children with autism or DD/LI.

Diagnostic standard: DSM-IV or DSM-IV-TR criteria were used to diagnose autism. In the second validation sample the Autism Diagnostic Observation Schedule-Generic (ADOS-G) was also used. Diagnostic evaluation was carried out by a multidisciplinary team including a psychologist and a speech/ language pathologist, who were blind to the STAT result in the initial study but not in the second study.

Outcomes: Sensitivity and specificity, positive and negative predictive value, false positive and false negative rate.

\section{MAIN RESULTS}

In the initial validation sample consisting of children with autism or $\mathrm{DD} / \mathrm{LI}$, the STAT yielded a sensitivity of 0.92 , a specificity of 0.85 , a positive predictive value of 0.86 , and a negative predictive value of 0.92 compared with DSM-IV diagnoses. In the second, larger validation sample, the STAT performed well at distinguishing between the ADOS-G classifications of autism and non-autistic spectrum. However, it was less accurate at distinguishing autism from pervasive developmental disorder not otherwise specified (see http://www.ebmentalhealth.com/supplemental for table).

\section{CONCLUSIONS}

Although the STAT has a high sensitivity and specificity in populations of children with autism or non-autistic spectrum disorders, it is less accurate at distinguishing children with autism from those with pervasive developmental disorder not otherwise specified.

For correspondence. Wendy Stone Vanderbilt Child Development Center 426 Medical Center South, 2100 Pierce Avenue, Nashville, TN 37232-373, USA; wendy.stone@vanderbilt.edu

Sources of funding: US Department of Education, National Institute of Mental Health, and National Institute of Child Health and Human Development.

\section{NOTES}

The STAT is intended to be used in a population of children already identified as having developmental problems. It was therefore calibrated in this study in a select population consisting only of children with autism or developmental delay/language impairment. The test's value is limited by its apparent lack of specificity in a more mixed population. However, if it is used as a screening test, rather than for definitive diagnoses, this may not be a serious flaw. The test's performance in the second sample may have been falsely enhanced because the result was known to those making the clinical diagnoses.

\section{Commentory}

This paper updates findings reported by Stone et al using a slightly different scoring system, and confirms that the Screening Tool for Autism in Two Year Olds (STAT) performs well as a second level screen for autism (that is, targeting children already identified due to developmental concerns). Using the cut-off score established and validated in this paper, the STAT was shown to have excellent agreement with the Autism Diagnostic Observation Scale (ADOS; autism cut-off) when comparing children with autism and language impairments or other developmental delays (only 2 of 52 children scoring in the STAT high risk range failed to exceed the ADOS threshold for autism), but children with a clinical diagnosis of pervasive developmental disorder, not otherwise specified (PDD-NOS) were more or less equally divided into the "high risk" and "low risk" categories by the STAT. The STAT is briefer and may be simpler to administer than the ADOS, and hence may be feasibly used to help service providers make more rational decisions when assigning clinical referrals to specialised autism assessment teams versus more general developmental clinics. Given that current intake processes at developmental centres often make these decisions informally, the potential utility of a second level screen such as the STAT might best be evaluated in a randomised controlled trial, with a focus on clinically relevant outcomes such as length of time from the point of referral to diagnosis.

The STAT is not the only screening tool to show modest sensitivity in detecting children with PDD-NOS; for example the Checklist for Autism in Toddlers (CHAT) has extremely low sensitivity for this diagnostic subtype.' However, children with PDD-NOS comprise a substantial proportion of the total number of children on the autistic spectrum in recent epidemiological studies. ${ }^{2} 3$ The sometimes subtle and heterogeneous manifestations of PDD-NOS clearly present a challenge to detection via screening, even with an observational tool such as the STAT. Lonnie Zwaigenbaum MD, MSc Associate Professor, Department of Pediatrics, Faculty of Health Sciences, McMaster University, Hamilton, Ontario, Canada

1 Baird G, Charman T, Baron-Cohen S, et al. A screening instrument for autism at 18 months of age: a 6-year follow-up study. I Am Acad Child Adolesc Psychiatry 2000;39:694-702.

2 Bertrand J, Mars A, Boyle C, et al. Prevalence of autism in a United States population: the Brick Township, New Jersey investigation. Pediatrics 2001;108:1155-61.

3 Chakrabarti S, Fombonne E. Pervasive developmental disorders in preschool children. JAMA 2001;285:3141-2. 